Supporting Information

\title{
Interference of Solvatochromic Twist in Amyloid Nanostructure for Light-Driven Biocatalysis
}

\author{
Giyeong Son ${ }^{\dagger}$ Jinhyun Kimץ, and Chan Beum Park ${ }^{*}$
}

Department of Materials Science and Engineering, Korea Advanced Institute of Science and Technology (KAIST), 335 Science Road, Daejeon 305-701, Republic of Korea

*E-mail: parkcb@kaist.ac.kr

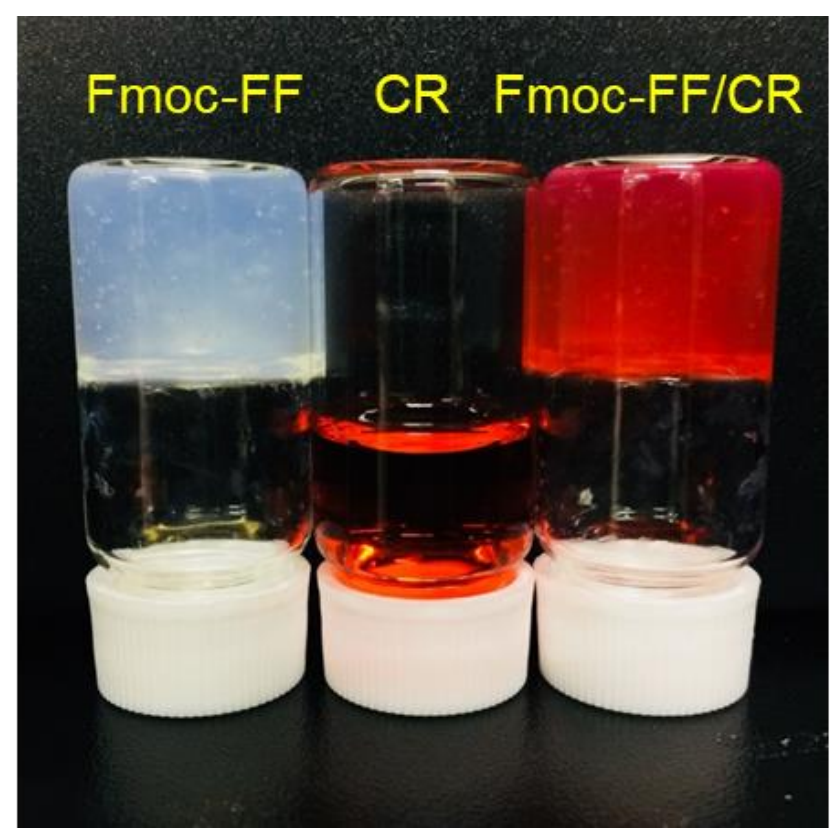

Figure S1. Photographs of CR solution, Fmoc-FF, and Fmoc-FF/CR hydrogels. The FmocFF hydrogel was white, while the Fmoc-FF/CR hydrogel was red. The concentrations of Fmoc-FF and CR were $10 \mathrm{mg} \mathrm{ml}^{-1}$ and $200 \mu \mathrm{M}$, respectively. 

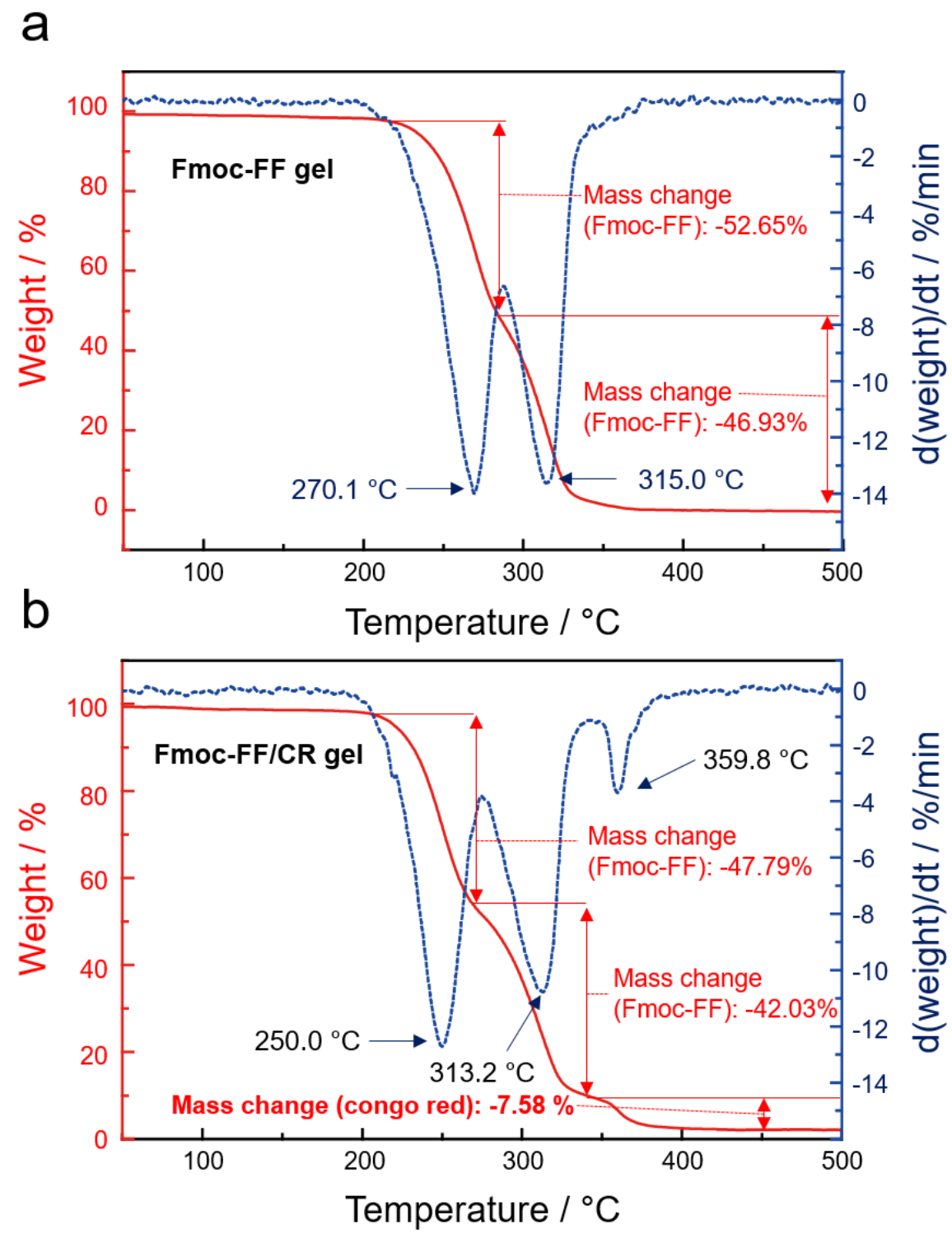

Figure S2. Thermogravimetric analysis of (a) Fmoc-FF and (b) Fmoc-FF/CR hydrogels. These two hydrogels were lyophilized before the analysis. The weight loss of the pristine Fmoc-FF hydrogel occurred from 200 to $350{ }^{\circ} \mathrm{C}$. This thermal degradation range of the Fmoc-FF was similar in the Fmoc-FF/CR hydrogel; the CR dyes were thermally decomposed after $350{ }^{\circ} \mathrm{C}$. 


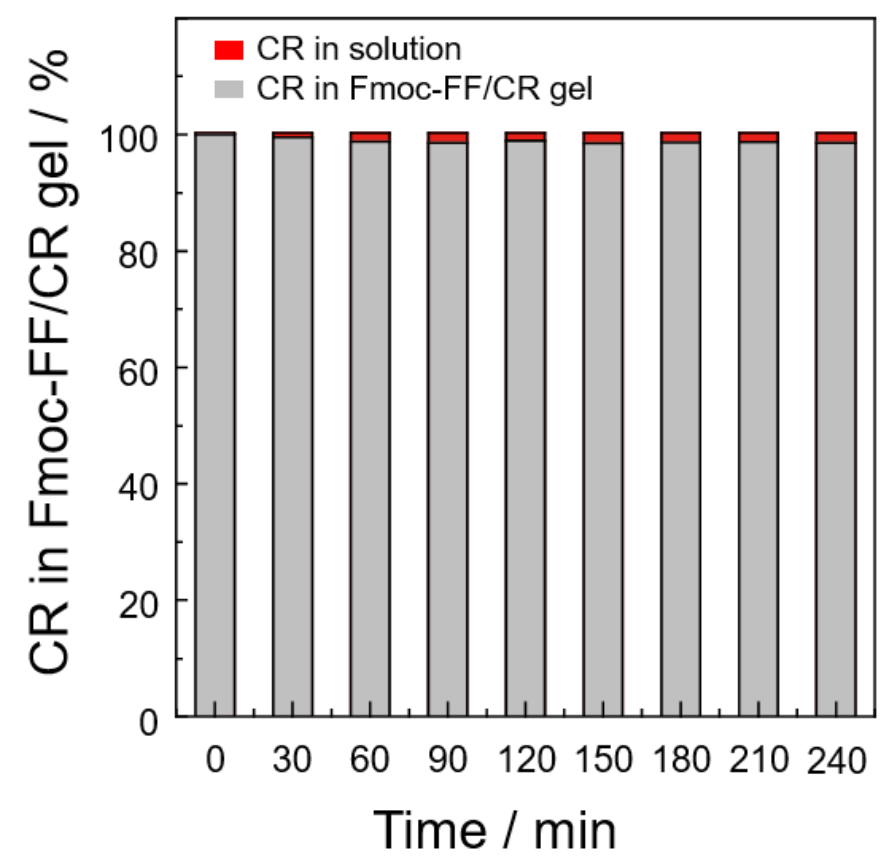

Figure S3. Time profile of the relative amount of CR in the supernatant and Fmoc-FF/CR hydrogel. Note that we injected deionized water above the Fmoc-FF/CR hydrogel and obtained the absorbance spectra of the supernatant to measure the concentration of free CR. Then, we estimated a relative amount of the CR in the Fmoc-FF/CR hydrogel by subtracting the amount of free CR in the solution from the initial amount of CR in Fmoc-FF/CR hydrogel. 


\section{a}

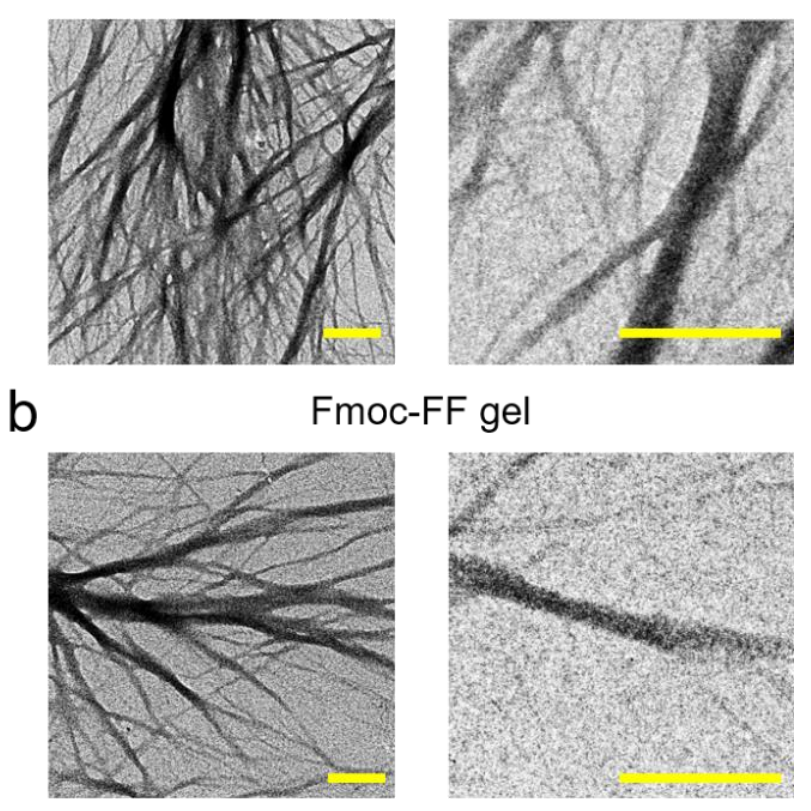

Fmoc-FF/CR gel

Figure S4. Transmission electron microscopy images of (a) Fmoc-FF and (b) Fmoc-FF/CR hydrogels. Scale bar: $500 \mathrm{~nm}$.

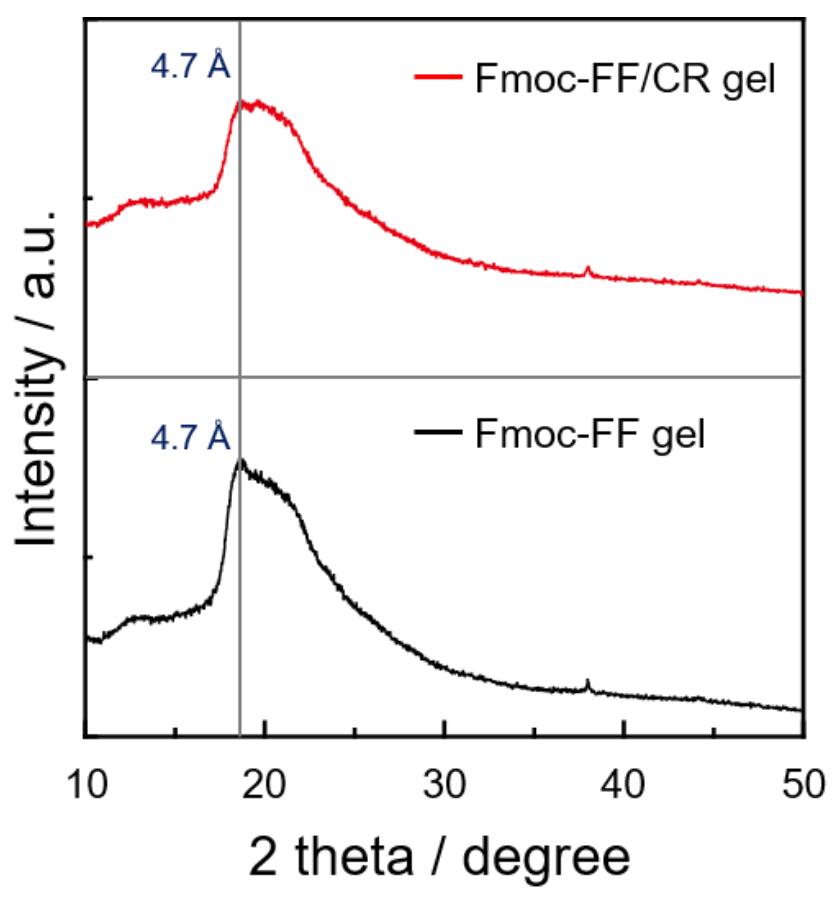

Figure S5. X-ray diffraction patterns of Fmoc-FF and Fmoc-FF/CR hydrogels. $\mathrm{Cu} \mathrm{K} \alpha 1$ radiation wavelength: $1.5406 \AA$. The characteristic peak at $18.65^{\circ}$ (d: $4.7 \AA$ ) stemmed from the interstrand distance of Fmoc-FF nanofibers (Biomacromolecules 2017, 18, 3551-3556). 


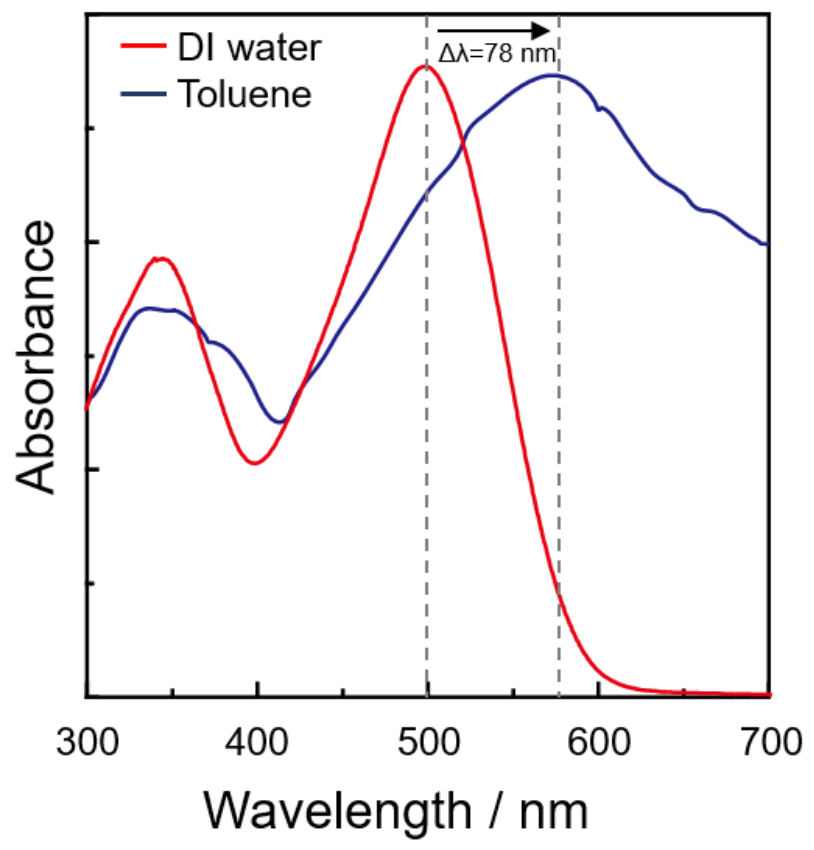

Figure S6. Absorbance spectra of the CR in deionized water and toluene to investigate the solvatochromic effect of free CR. The concentration of CR was $100 \mu \mathrm{M}$.

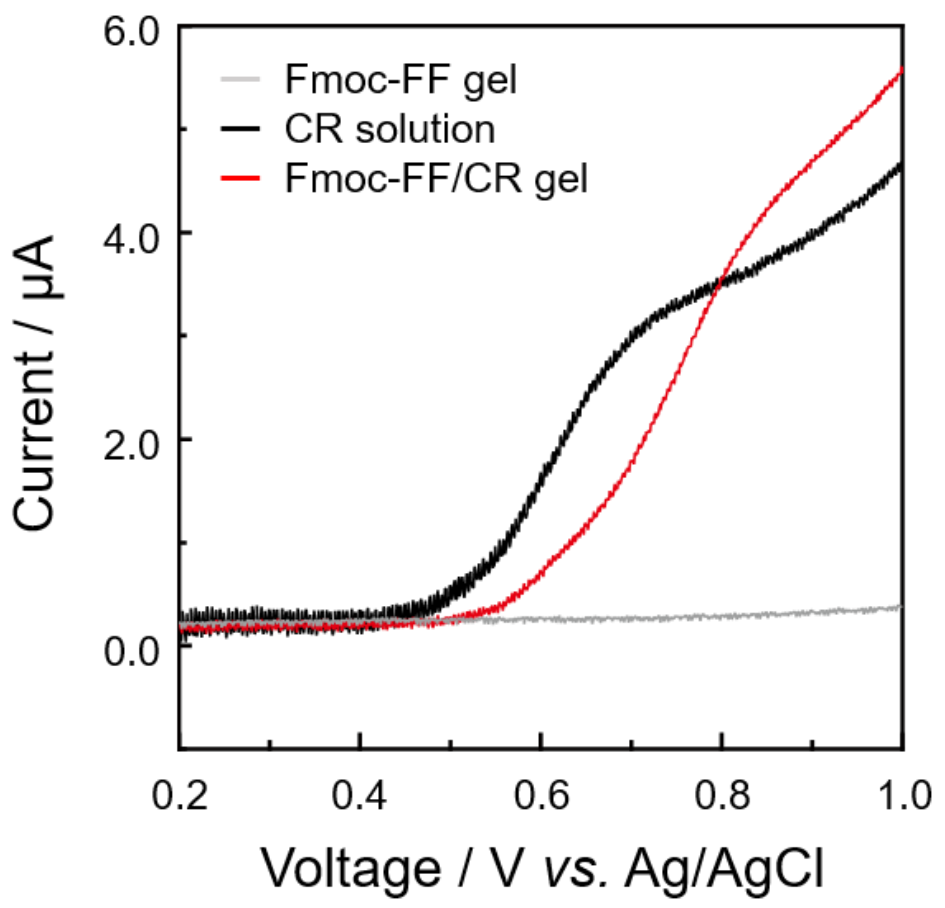

Figure S7. Linear sweep voltammograms of CR solution, Fmoc-FF and Fmoc-FF/CR hydrogels. Scan rate: $50 \mathrm{mV} \mathrm{s}^{-1}$. The working electrodes of Fmoc-FF and Fmoc-FF/CR hydrogels were Fmoc-FF-coated ITO and Fmoc-FF/CR-coated ITO, respectively. 
a

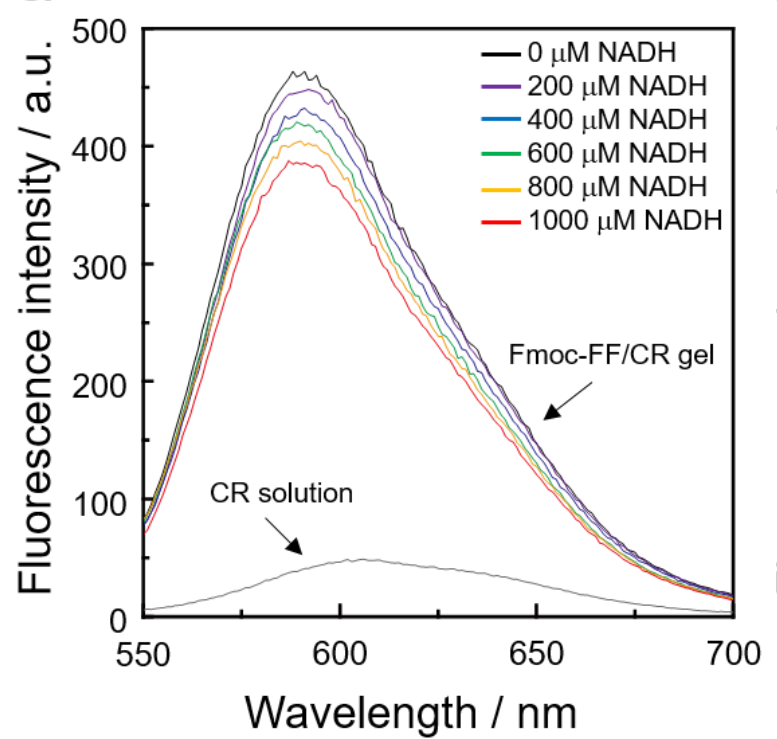

b

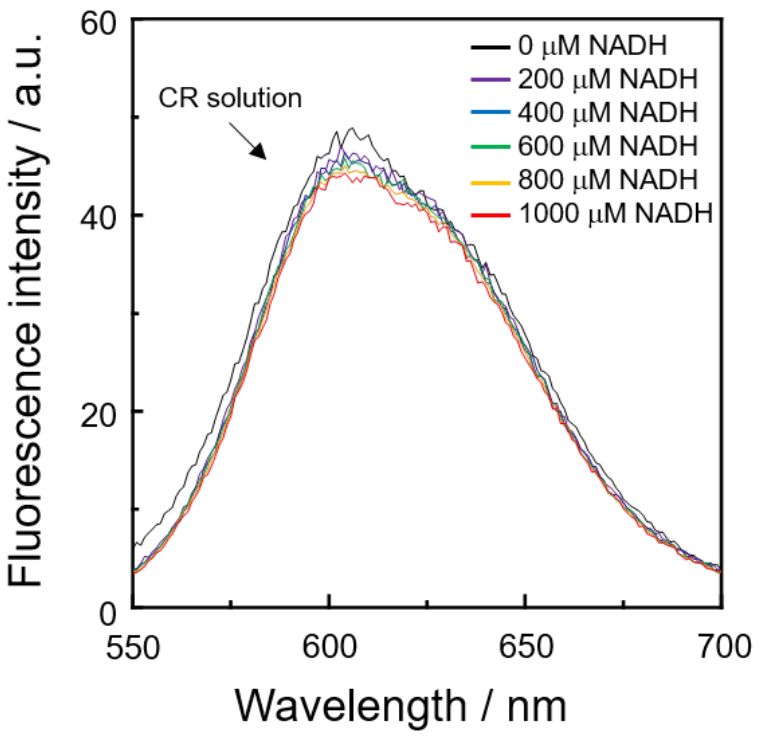

Figure S8. Fluorescence spectra of (a) Fmoc-FF/CR hydrogel and (b) CR solution at different concentrations of NADH. Excitation wavelength: $500 \mathrm{~nm}$.

a

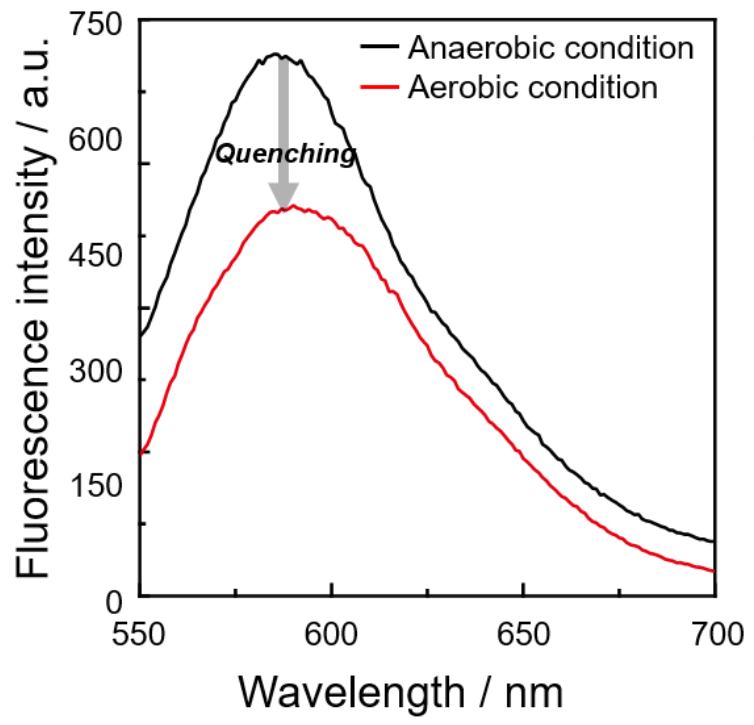

b

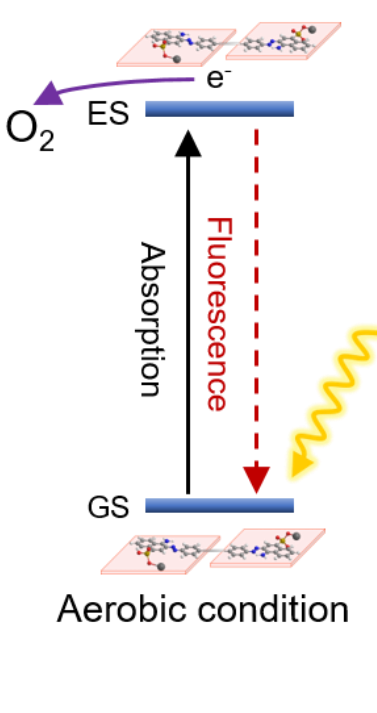

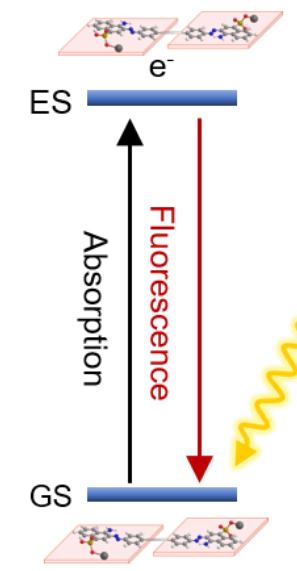

Anaerobic condition

Figure S9. (a) Fluorescence spectra of Fmoc-FF/CR hydrogel under anaerobic or aerobic conditions. Excitation wavelength: $500 \mathrm{~nm}$. The anaerobic solution was prepared by purging with Ar gas. (b) Illustration of an oxidative quenching of Fmoc-FF/CR hydrogel by $\mathrm{O}_{2}$ molecules under light irradiation. 


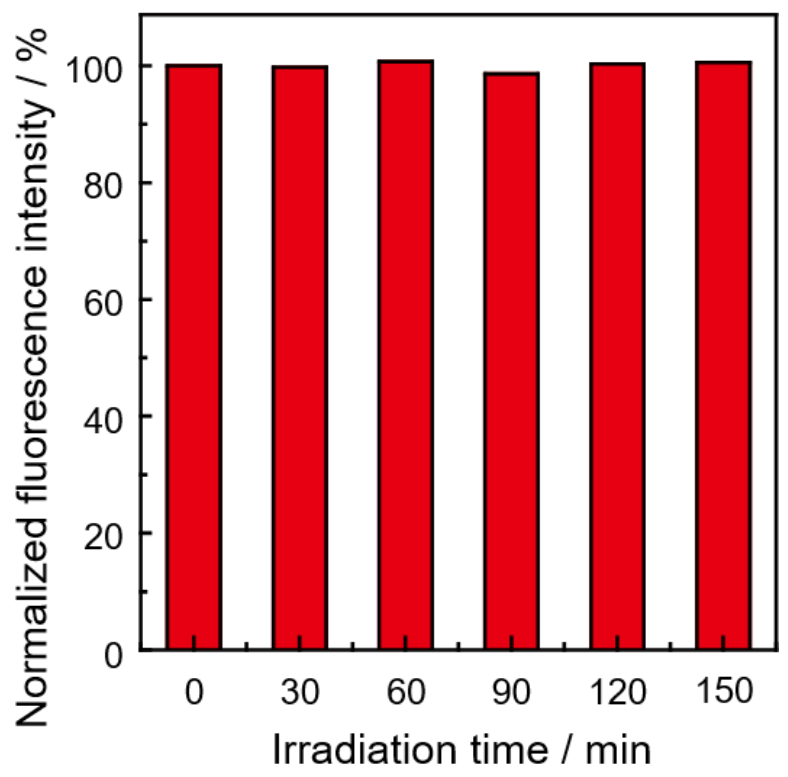

Figure S10. Time profile of normalized fluorescence intensity $\left(I / I_{0}\right)$ of Fmoc-FF/CR hydrogel during photobiocatalytic oxidation. Excitation wavelength: $500 \mathrm{~nm}$. Emission wavelength: $600 \mathrm{~nm}$. Reaction condition: $10 \mathrm{mg} \mathrm{ml}^{-1}$ Fmoc-FF, $200 \mu \mathrm{M} \mathrm{CR}, 5 \mathrm{U} \mathrm{ml}^{-1}$ $\mathrm{ScADH}, 2 \mathrm{mM} \mathrm{NADH}$, and $10 \mathrm{mM}$ ethanol in a sodium phosphate buffer $(25 \mathrm{mM}, \mathrm{pH} 8.8)$.

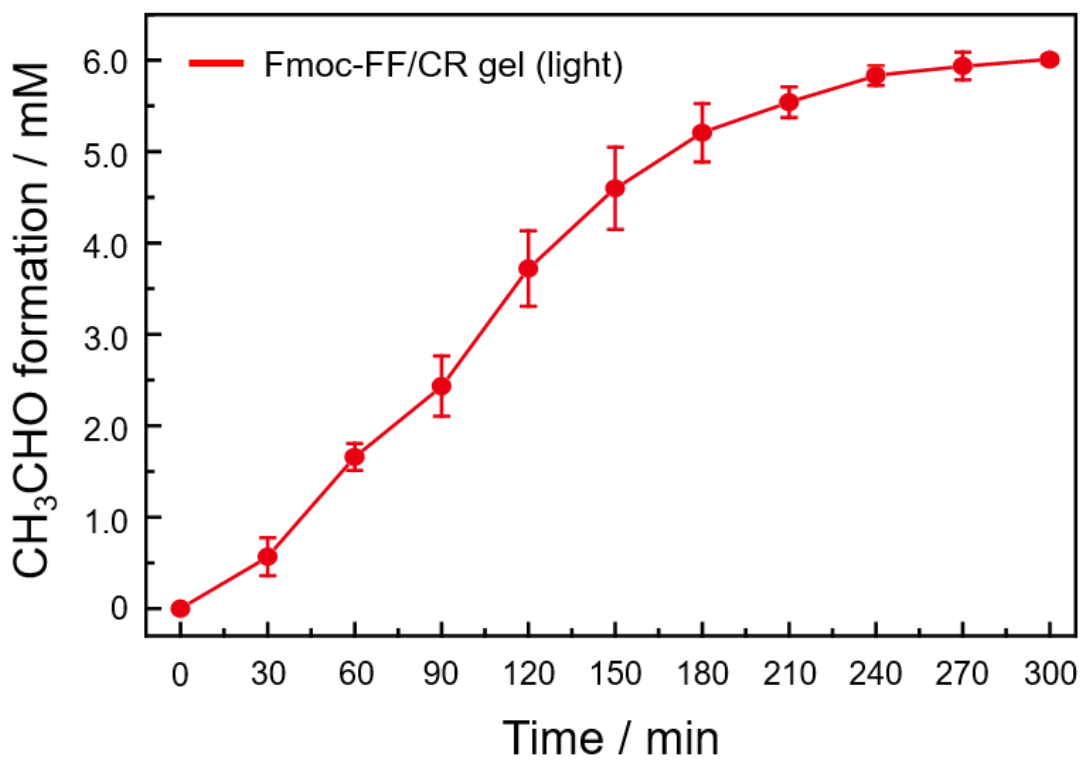

Figure S11. Time profile of photoenzymatic production by Fmoc-FF/CR hydrogel with ScADH. Reaction condition: $10 \mathrm{mg} \mathrm{ml}^{-1}$ Fmoc-FF, $200 \mu \mathrm{M} \mathrm{CR}, 5 \mathrm{U} \mathrm{ml}^{-1}$ ScADH, $2 \mathrm{mM}$ $\mathrm{NADH}$, and $10 \mathrm{mM}$ ethanol in a sodium phosphate buffer $(25 \mathrm{mM}, \mathrm{pH} 8.8)$. 